\title{
ESPECIES DE NEOHYDATOTHRIPS JOHN (THYSANOPTERA: THRIPIDAE) DE NAYARIT, MÉXICO, Y DESCRIPCIÓN DE UNA ESPECIE NUEVA
}

\author{
Rita D. VALENZUELA-GARCÍA, ${ }^{1}$ AXel P. RETANA-SALAZAR, ${ }^{2}$ Roberto \\ M. JOHANSEN-NAIME, ${ }^{3}$ OSWALdo GARCÍA-MARTÍNEZ, ${ }^{1}$ GABRIEL \\ GALLEGOS-MORALES ${ }^{1}$ \& CARLOS CARVAJAL-CAZOLA ${ }^{4}$ \\ ${ }^{1}$ Universidad Autónoma Agraria Antonio Narro (Departamento de Parasitología), Saltillo, Coahuila, \\ México. C.P. 25315.<rita.dinora@gmail.com> \\ ${ }^{2}$ Programa Universitario de desarrollo Agrario (PUA), Centro de Investigación en Estructuras \\ Microscópicas (CIEMic), Ciudad de la Investigación, Universidad de Costa Rica. San Jose, Costa \\ Rica.<apretana@gmail.com> \\ ${ }^{3}$ Instituto de Biología, Universidad Nacional Autónoma de México (IBUNAM), México DF, México. \\ $<$ naime@ibiologia.unam.mx $>$ \\ ${ }^{4}$ Unidad Académica de Agricultura, Universidad Autónoma de Nayarit, Xalisco, Nayarit, México. Km- \\ 9 Carretera Tepic-Compostela. C.P. 63190.<carvajal@nayar.uan.mx>
}

Valenzuela-García, R. D., A. P. Retana-Salazar, R. M. Johansen-Naime, O. García-Martínez, G. Gallegos-Morales, C. Carvajal-Cazola. 2012. Especies de Neohydatothrips John (Thysanoptera: Thripidae) de Nayarit, México, y descripción de una especie nueva. Acta Zoológica Mexicana (n. s.), 28(1): 49-55.

RESUMEN. Se dan a conocer las especies de Neohydatothrips del estado de Nayarit, asociadas a diferentes cultivos, y se describe una nueva especie cercana a $N$. mirandai Johansen, la cual se diferencia por tener el pronoto de color amarillo.

Palabras clave: Phaseolus vulgaris, Mangifera indica, Solanum lycopersicum, San Blas, Santiago Ixcuintla.

Valenzuela-García, R. D., A. P. Retana-Salazar, R. M. Johansen-Naime, O. García-Martínez, G. Gallegos-Morales, C. Carvajal-Cazola. 2012. Neohydatothrips John species (Thysanoptera: Thripidae) in Nayarit, Mexico, and description of a new species. Acta Zoológica Mexicana (n. s.), 28(1): 49-55.

ABSTRACT. The species of Neohydatothrips from the state of Nayarit, associated to different crops are presented. Also a new species similar to $N$. mirandai Johansen is described which differs from the rest by having a yellow pronotum.

Key words: Phaseolus vulgaris, Mangifera indica, Solanum lycopersicum, San Blas, Santiago Ixcuintla.

Recibido: 16/11/2010; aceptado: 05/10/2011. 


\section{INTRODUCCIÓN}

El género Neohydatothrips John comprende 80 especies en el mundo (Nakahara 1988), siendo muy similares en apariencia general y detalles de estructura, a las de Hydatothrips Karny. Los dos géneros pueden separarse por la morfología del metasterno, que en las especies de Neohydatothrips está ligeramente redondeado en el borde anterior (Mound \& Marullo 1996).

Mound y Marullo (1996) describen 13 especies de Neohydatothrips para Centro América, y Stannard (1968) registra 11 para Illinois, Estados Unidos de América. En México, en este género Johansen y Mojica (2007) reconocen cinco especies de interés fitosanitario y Cambero et al. $(2009,2010)$ registran cuatro especies fitófagas en huertos de aguacate (Persea americana) de Nayarit.

El presente trabajo deriva de la investigación titulada "Especies de thrips (Thysanoptera) presentes en cultivos y arvenses en Nayarit, México" donde los objetivos fueron: identificar las especies de thrips asociadas a seis cultivos y arvenses; determinar los índices de diversidad de thrips en los diferentes cultivos y arvenses. Para lo cual se planteo el siguiente objetivo para el presente trabajo: identificación de especies de género Neohydatothrips.

\section{MATERIALES Y MÉTODOS}

Los muestreos para obtener adultos de thrips se realizaron semanalmente de noviembre del 2009 a febrero de 2010, en los municipios de San Blas y Santiago Ixcuintla ubicados en la región costera del estado de Nayarit, utilizando red entomológica de golpeo con la cual se daban 100 redazos al azar en plantas de tomate (Solanum lycopersicum L.), frijol (Phaseolus vulgaris L.), árboles de mango (Mangifera indica L.) y arvenses aledañas a estas especies vegetales; se eligieron estos cultivos debido a su importancia tanto por superficie como por producción. Los ejemplares de thrips recolectados se colocaron en frascos de plásticos con alcohol al $70 \%$ debidamente etiquetados y posteriormente, en el laboratorio de taxonomía de adultos del Departamento de Parasitología Agrícola (DPA) de la Universidad Autónoma Agraria Antonio Narro (UAAAN), se montaron, utilizando la técnica descrita por Johansen \& Mojica (1997). Para la identificación taxonómica se utilizaron las claves de Stannard (1968), Mound \& Marullo (1996), realizando esta actividad en el DPA-UAAAN; la posterior confirmación se realizó en el Centro de Investigación en Estructuras Microscópicas (CIEMic) de la Universidad de Costa Rica, el material de referencia y la nueva especie fueron depositados en la colección del Instituto de Biología de la Universidad Nacional Autónoma de México (UNAM) 


\section{RESULTADOS}

Durante los cuatro meses de muestreo, se recolectó un total de 1232 thrips adultos y ninfas, montando 751 adultos en buenas condiciones, de los cuales, 63 especímenes correspondieron al género Neohydatothrips (Cuadro 1).

Neohydatothrips basilaris es nuevo registró para el estado de Nayarit. A continuación se describe una especie nueva de Neohydatothrips para el occidente de México, que se recolectó en frijol (P. vulgaris L.).

\section{Neohydatothrips angelorum sp. nov.}

Holotipo hembra macróptera. México, Nayarit, San Blas, en la localidad de Guadalupe Victoria en Phaseolus vulgaris L., 6 msnm, 21 ${ }^{\circ} 42^{\prime} 47^{\prime \prime}$ ', latitud Norte, 115 $12^{\prime} 29^{\prime \prime}$ longitud Oeste. Rita Dinora Valenzuela García col. 17 de diciembre 2011.

Medidas del Holotipo en micras. Cuerpo completo 1275. Antenómeros: $\mathrm{I}=25$, $\mathrm{II}=37.5, \mathrm{III}=52.5, \mathrm{IV}=60, \mathrm{~V}=50, \mathrm{VI}=52.5, \mathrm{VII}=12.5$, VIII=17.5. Setas posteroculares 25 . Alas 760 . Fémures: $\mathrm{I}=165$ de largo por 55 de ancho, $\mathrm{II}=160$ de largo por 40 , $\mathrm{III}=225$ de largo por 45 de ancho.

Diagnosis. Alas bandeadas, con el ápice pálido, dos sedas en la segunda vena. Mesonoto estriado, dos pares de sedas medias no alineadas.

Coloración bicolor (Fig. 1). Cabeza castaño oscuro, pronoto amarillo (Fig. 2), mesotórax castaño oscuro, parte lateral del metatórax y segmento abdominal I castaño claro, segmentos II-III castaño oscuro, del IV-VI amarillos, VII-VIII castaño oscuro, IX-X amarillo. Antenómeros I-III amarillos, IV-V castaño con base amarilla, VI-VIII castaño. Tibias I-II pálidas, fémur y tarsos amarillos. Tibias III amarillas con ápice castaño.

Cuadro 1. Especies del género Neohydatothrips recolectadas, en tres cultivos y arvenses en Nayarit, México.

\begin{tabular}{lccccc}
\hline \multicolumn{1}{c}{ Especies } & Tomate & Frijol & Mango & Arvenses & Total \\
\hline Neohydatothrips gracilipes (Hood, 1924) & 3 & 19 & 1 & 11 & 34 \\
Neohydatothrips basilaris (Hood, 1941) & - & - & - & 1 & 1 \\
Neohydatothrips inversus (Hood, 1928) & 1 & - & - & - & 1 \\
Neohydatothrips signifer (Priesner, 1932) & 1 & 27 & - & 8 & 36 \\
Neohydatothrips angelorum sp. nov. & - & 1 & - & - & - \\
Total & 5 & 47 & 1 & 20 & 62 \\
\hline
\end{tabular}




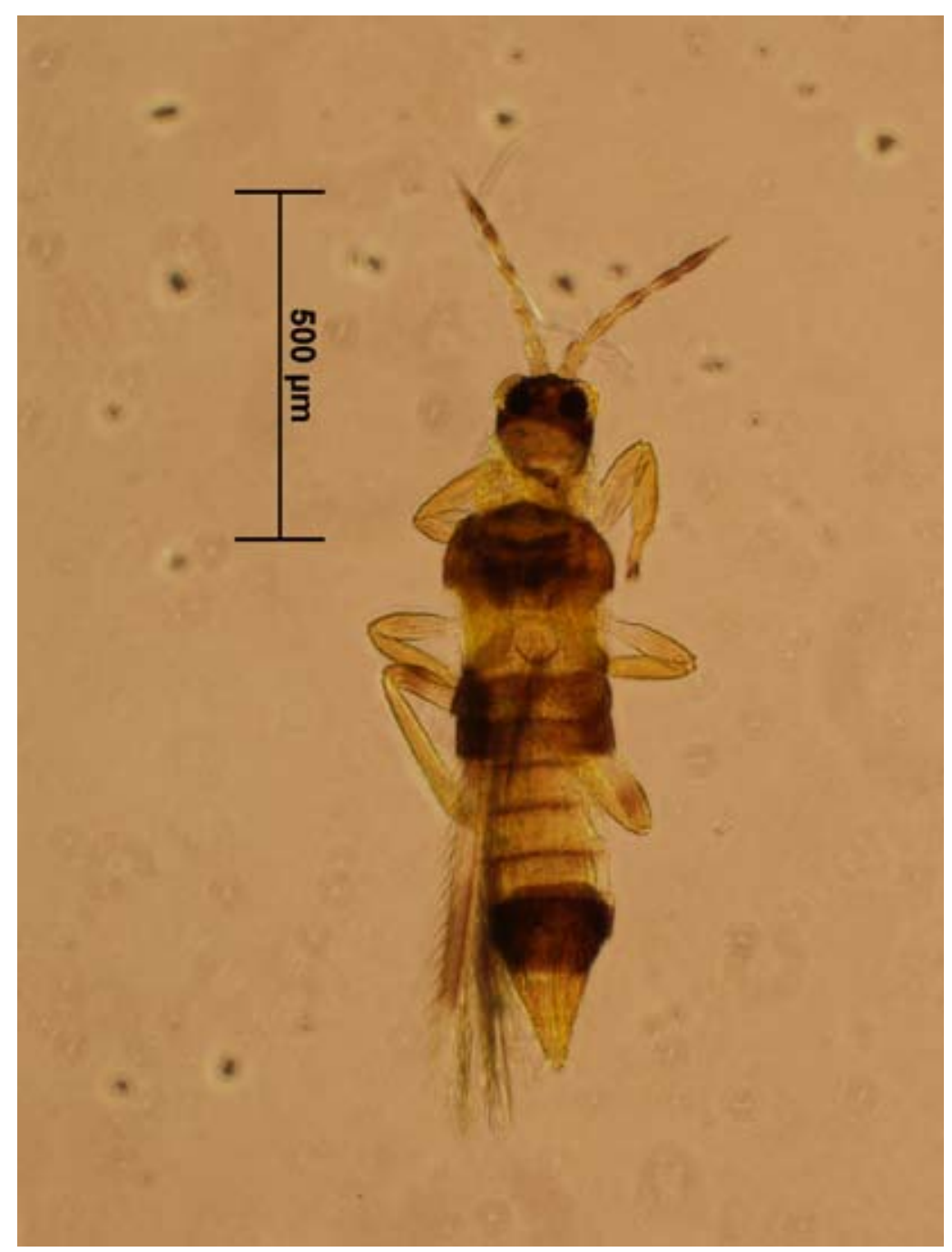

Fig. 1.

Cabeza. Sedas ocelares largas, seda ocelar III en el margen del triángulo ocelar detrás del ocelo I; triángulo ocelar estriado transversalmente. Apodema claramente separado del margen de los ojos, con cinco pares de sedas.

Tórax. Pronoto con reticulación transversal en los márgenes anterior y posterior, estriado transversalmente en apodema con cuatro pares de sedas. Metanoto estriado transversal y longitudinalmente en la parte media. Escama con 5 sedas. 


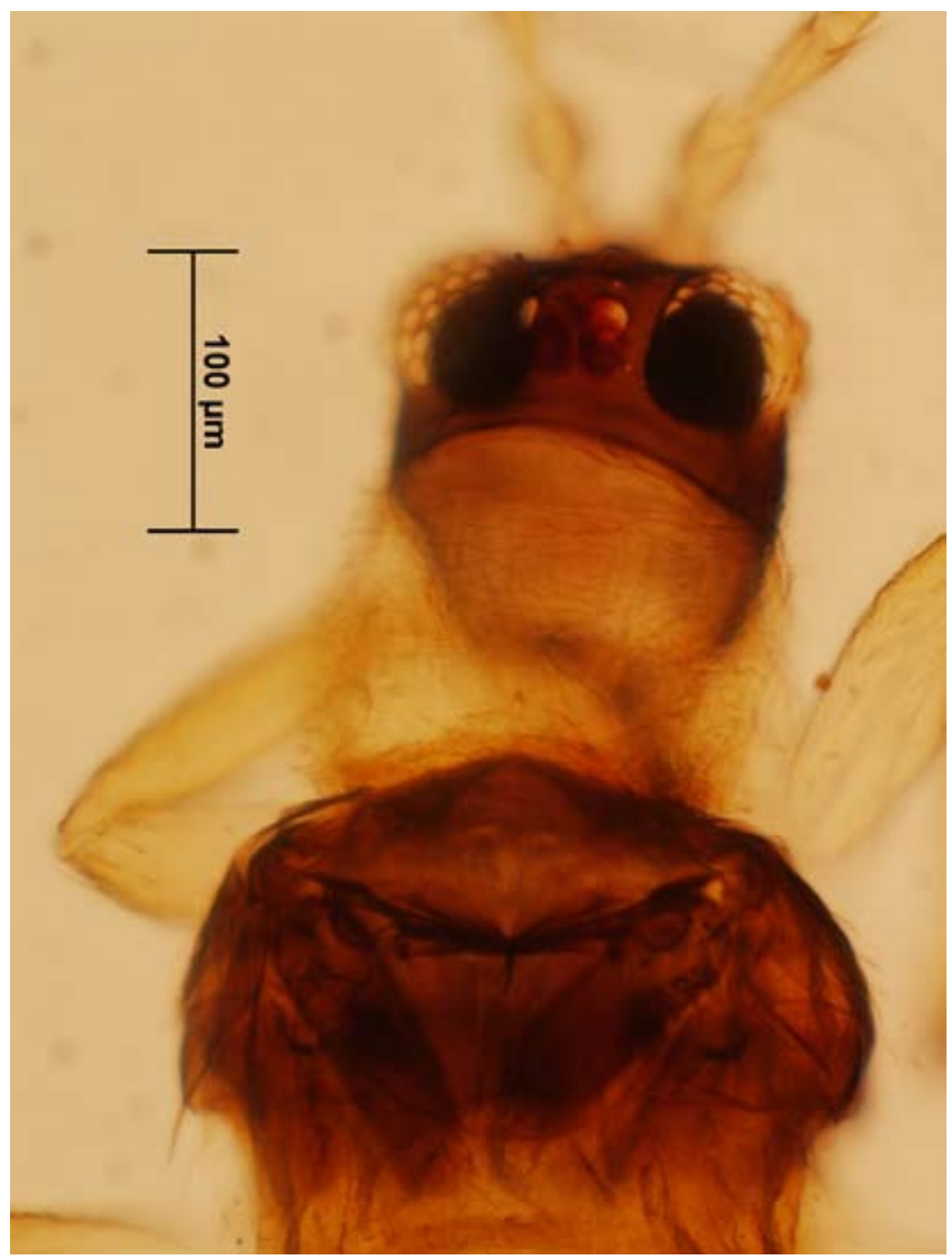

Fig. 2.

Abdomen. Segmentos abdominales con microtriquias en el tercio lateral. Segmentos II-VI con peine lateral y VII-VIII con peine completo de microtriquias; además, tiene microtriquias en la parte media. Tergito VIII con un par de sensilias campaniformes minúsculos en medio de las sedas mediales.

Etimología. El nombre de la especie, angelorum, proviene del nombre propio del hijo de la autora José Ángel A. Valenzuela y del apellido de Omar García Ángel, 
a quienes se dedica esta especie en reconocimiento a su influencia en el desarrollo académico de la misma.

\section{DISCUSIÓN}

La última revisión de las especies de este género en el Neotrópico (Mound \& Marullo 1996), explicita que solo $N$. pseudoannulipes Johansen, $N$. flavicollis Hood, $N$. mirandai Johansen y $N$. tibialis Johansen presentan patrón de coloración de las alas bandeado. De estas, N. pseudoannulipes se segrega fácilmente por la escasa esculturación de la región interocelar, mientras que las demás presentan un claro patrón de estriaciones en esta sección de la cabeza. En la especie nueva, este patrón es similar al de las especies del grupo tibialis, con el apodema occipital alejado de los ojos; $N$. angelorum sp. nov., se distingue de tibialis por el patrón de coloración bicolor que presenta y que la acerca a $N$. mirandai, descrita por Johansen (1981), para México y puede diferenciarse claramente de esta, por presentar un patrón de coloración similar, pero con el pronoto amarillo, mientras que $N$. mirandai lo presenta castaño, según la descripción original (Johansen 1981). Algunos especialistas consideran que las variaciones en coloración no son importantes, pero hallazgos recientes demuestran lo contrario en grupos de insectos, como las mariposas, donde el aislamiento reproductivo puede reflejarse en cambios de patrones de coloración (Jiggins et al. 2001). Los análisis de filogenia en Frankliniella indican que muchos patrones de coloración presentan una alta estructuración con los patrones de cladogénesis (Retana 2010), lo que sugiere que estas características son de valor en la segregación de especies en insectos. La importancia de estas especies para la agricultura radica en que son especies fitófagas que pueden llegar a ocasionar perdidas en la producción de diferentes cultivos.

\section{LITERATURA CITADA}

Cambero, O. J, R. M. Johansen, O. García, C. R. Carvajal, N. Isiordia, M. Cantu. 2009. Thrips fitófagos en huertas de aguacate cv. Hass en Nayarit, México. Brenesia, 71-72: 61-64.

Cambero, O. J, R. M. Johansen, O. García, C.R. Carvajal, N. Isiordia, M. Cantu. 2010. Thrips (Thysanoptera) del aguacate (Persea americana) en Nayarit, México. Revista Colombiana de Entomología, 36: 47-51.

Jiggins, C. D., R. E. Naisbit, R. L. Coe, \& J. Mallet. 2001. Reproductive isolation caused by colour pattern mimicry. Nature, 411: 302-305.

Johansen, R. M. 1981. Seis nuevos Tisanópteros (Terebrantia: Heterothripidae; Thripidae) de Chiapas, México. Anales del Instituto de Biología, Universidad Nacional Autónoma de México. 50 [1979]: 159-178.

Johansen, R. M., \& G. A. Mojica. 1997. Importancia agrícola de thrips, pp: 11-18. In: Manual sobre Entomología y Acarología Aplicada. Memorias del Seminario Introducción a la Entomología y Acarología Aplicada. Mayo 22 al 24. UAEP SME. 
Johansen, R. M y G. A. Mojica. 2007. Acerca de unos ensambles de especies de trips, en árboles de aguacate (Persea americana Mill.), en México, pp. 1-8. In: Proceedings VI World Avocado Congress (Actas VI Congreso Mundial del Aguacate), Viña Del Mar, Chile.

Mound, L. A., \& R. Marullo. 1996. The Thrips of Central and South America: An Introduction. Memoirs on Entomology International, 6: 1-488.

Nakahara, S. 1988. Generic assignments of North American species currently assigned to the genus Sericothrips Haliday (Thysanoptera: Thripidae). Proceedings of the Entomological Society of Washington, 90: 480-483.

Retana, A. P. 2010. El grupo genérico Frankliniella: el significado filogenético de sus principales caracteres morfológicos (Thysanoptera: Thripidae; Thripini). Métodos en Ecología y Sistemática, 5: 1-22.

Stannard, L. J. 1968. The Thrips, or Thysanoptera, of Illinois. Bulletin of the Illinois Natural History Survey, 29: 213-552. 